\title{
MicroRNA-124-5p delays the progression of cerebral aneurysm by regulating FoxO1
}

\author{
RU-KE WANG ${ }^{1}$, YUAN-YUAN SUN ${ }^{2}$, GUANG-YOU LI ${ }^{1}$, HUA-TANG YANG ${ }^{1}$, \\ XIU-JIE LIU ${ }^{1}$, KE-FENG LI ${ }^{1}, \mathrm{XU} \mathrm{ZHU}^{1}$ and GUO-YUAN YU ${ }^{1}$ \\ ${ }^{1}$ Section 2, Department of Neurosurgery, Handan Central Hospital, Handan, Hebei 056001; \\ ${ }^{2}$ CT Room, Handan First Hospital, Handan, Hebei 056002, P.R. China
}

Received August 20, 2020; Accepted March 17, 2021

DOI: $10.3892 / \mathrm{etm} .2021 .10606$

\begin{abstract}
Cerebral aneurysm (CA) is a common brain disease, and the development of cerebral aneurysm is driven by inflammation and hemodynamic stress. MicroRNA (miR)-124-5p is reported to be associated with inflammatory response in brain disease such as cerebral ischemia-reperfusion injury. However, the function and molecular mechanism of miR-124-5p in CA are not clear, thus, the effects of miR-124-5p on inflammatory response in CA were explored. Firstly, the expression of miR-124-5p in the peripheral blood of patients with CA and the control group was detected by reverse transcription-quantitative PCR. Then, the human umbilical vein endothelial cells (HUVECs) were used as an in vitro model system and stimulated with interleukin (IL)- $1 \beta$ to simulate the inflammatory environment of CA, and the expression of miR-124-5p was detected. Next, the effect of miR-124-5p on the migration and invasion of HUVECs was detected using Transwell assays. Meanwhile, the function of miR-124-5p on various inflammatory factors was determined by western blotting and enzyme-linked immunosorbent assay (ELISA). Next, the TargetScan website was used to predict FoxO1 as a target gene of miR-124-5p, and this target association was validated by double luciferase reporter assay and western blotting. Finally, the interaction of miR-124-5p with FoxO1 in CA was measured by Transwell western blotting and ELISA assays. The results showed that the expression level of miR-124-5p in the peripheral blood of patients with CA was lower compared with that of control group, and the miR-124-5p in HUVECs stimulated by IL-1 $\beta$ was less compared with that in normal HUVECs. Besides, miR-124-5p could inhibit the migration and invasion abilities of HUVECs and the release of inflammatory factors. Additionally,
\end{abstract}

Correspondence to: Dr Guo-Yuan Yu, Section 2, Department of Neurosurgery, Handan Central Hospital, 59 Congtai North Road, Congtai, Handan, Hebei 056001, P.R. China

E-mail: yuguoyuan888_hch@163.com

Key words: cerebral aneurysm, microRNA-124-5p, FoxO1, interleukin-1 $\beta$ the overexpression of miR-124-5p was able to inhibit the expression of FoxO1. miR-124-5p-inhibitor promoted the migration and invasion of HUVECs, as well as inflammatory response, which was weakened following the introduction of FoxO1 small interfering RNA. Overall, the present study demonstrated that miR-124-5p could prevent the occurrence and development of cerebral aneurysm by downregulating the expression of FoxO1.

\section{Introduction}

Cerebral aneurysm (CA) is a cystic dilation of cerebral vascular wall caused by congenital or postnatal factors $(1,2)$. $\mathrm{CA}$ is characterized by the destruction of the integrity of arterial wall, endothelial dysfunction and extracellular matrix disorder (3). There are reports that $20-50 \%$ of patients with CA will experience the rupture of CA, leading to subarachnoid hemorrhage with high disability rate, high mortality rate and poor prognosis (4-7). Unfortunately, most patients are painless and asymptomatic when the aneurysm is not ruptured, which is a challenge for early treatment (8). At present, the occurrence and rupture of CA is closely associated with inflammation (9), and macrophages are the key effector cells (10). Monocyte chemoattractant protein-1 (MCP-1) induces macrophages to infiltrate CA walls and stimulates macrophages to differentiate into inflammatory phenotypes, thus affecting the formation and rupture of CA (11). In addition, the low wall shear stress of the aneurysm wall induces the continuous expression of MCP-1 in vascular smooth muscle cells $(12,13)$. However, the pathogenesis of aneurysm remains to be explored. Therefore, it is urgent to identify useful biomarkers for the diagnosis and prognosis of CA.

MicroRNAs (miRNAs) are shortstrands of noncoding RNAs composed of 20-25 nucleotides $(14,15)$. miRNAs can inhibit the expression of target genes by binding with the 3'-untranslated region (UTR) region of target $m R N A$ to inhibit the translation of mRNA or promote its degradation, thus regulating most biological processes such as cellular proliferation, migration, invasion and apoptosis (16-19). The dysregulation of miRNAs is frequently found in the pathogenesis of inflammatory diseases (20). For example, Kawano and Nakamachi (21) found that miR-124 can regulate the process of rheumatoid arthritis, and researchers also confirmed that the inhibition 
of miR-124 can activate the inflammatory response of retina and central neurons $(22,23)$. The formation and rupture of CA are attributed to the inflammatory dysfunction of endothelial cells, and some researchers have confirmed that miRNAs are associated with the formation of CA $(24,25)$. Therefore, it is speculated that miRNAs are involved in the inflammatory process of CA. It has been found that miR-124-5p can regulate macrophage phagocytosis and inhibit inflammatory pathways $(26,27)$. In addition, miR-124-5p has been shown to regulate angiogenesis (27). However, the role and molecular mechanism of miR-124-5p in CA has not been confirmed. The present study aims to explore the effect and the molecular mechanism of miR-124-5p on the occurrence and development of CA.

\section{Materials and methods}

Clinical samples. Peripheral blood samples from 30 patients with CA and 30 healthy individuals were collected from the Handan Central Hospital between August 2018 and January 2020. Before surgery, all patients did not receive treatment. Written informed consent was obtained from all participants and their families. The institutional ethics committee of Handan Central Hospital has approved this study on August 20, 2018.

In vitro CA model construction. Human umbilical vein endothelial cells (HUVECs) were chosen as the model cells in the present study, since it is a common cell in vascular system in vitro research, and are easier to obtain compared with other endothelial cells (28). In addition, the cellular function of HUVECs inhibited by inflammatory smooth muscle cells that are associated with CA (28). HUVECs purchased from Thermo Fisher Scientific, Inc. (cat. no. C0155C), and the cells were cultured in $37^{\circ} \mathrm{C}$ with $5 \% \mathrm{CO}_{2}$ for 2 days in DMEM complete medium (Thermo Fisher Scientific, Inc.) containing $10 \%$ fetal bovine serum (FBS; Thermo Fisher Scientific, Inc.). HUVECs were plated in 24 -well plates $\left(3 \times 10^{5}\right.$ cells/well $)$ and treated with $10 \mathrm{ng} / \mathrm{ml} \mathrm{IL-1b}$ (Invitrogen; Thermo Fisher Scientific, Inc.) for 1 day at $37^{\circ} \mathrm{C}$ to establish the inflammatory model of CA.

Reverse transcription-quantitative $(R T-q) P C R$. TRIzol reagent (Invitrogen; Thermo Fisher Scientific, Inc.) was used to extract the total RNA according to the manufacturer's instructions. The cDNA was synthesized using the First Strand cDNA Synthesis kit at $55^{\circ} \mathrm{C}$ for 30 min (Thermo Fisher Scientific, Inc.). Then, the mixture system including SYBR Green qPCR Master mix, cDNA templates and primers were applied for qPCR according to standard methods $\left(95^{\circ} \mathrm{C}\right.$ for 3 min to carry on pre-denaturation; $95^{\circ} \mathrm{C}$ for $30 \mathrm{sec}$ to carry out denaturation; $55^{\circ} \mathrm{C}$ for $30 \mathrm{sec}$ to subject to annealing; and $72^{\circ} \mathrm{C}$ for $60 \mathrm{sec}$ to subject to elongation) to detect the expression of miR-124-5p or FoxO1. Finally, 1.5\% agarose gel was used to electrophorese and visualize the products of PCR. The relative expression was calculated by the $2^{-\Delta \Delta \mathrm{Cq}}$ method (29). U6 small nuclear RNA and GAPDH were used as the internal controls of miR-124-5p and FoxO1, respectively. The primer sequences were: miR-124-5p forward, 5'-CGCGTGTTCACA GCGGAC-3'; miR-124-5p reverse, 5'-AGTGCAGGGTCC
GAGGTATT-3'; U6 forward, 5'-GCTTCGGCAGCACAT ATACTAAAAT-3'; U6 reverse, 5'-CGCTTCACGAATTTG CGTGTCAT-3'; FoxO1 forward, 5'-GGCTGAGGGTTAGTG AGCAG-3'; FoxO1 reverse, 5'-AAAGGGAGTTGGTGAAAG ACA-3' (30); GAPDH forward, 5'-TCGACAGTCAGCCGC ATCTTCTTT-3'; and GAPDH reverse, 5'-GCCCAATACGAC CAAATCCGTTGA-3' (31).

Cell transfection. miR-124-5p-inhibitor, miR-124-5p-mimics, FoxO1-small interfering (si)RNA (si-FoxO1) and negative controls (inhibitor-NC, mimic-NC and si-NC) were designed and construct by Shanghai Gene Pharmaceutical Technology Comsenz Inc. In addition, the Lipofectamine ${ }^{\mathrm{TM}} 3000$ Transfection kit was purchased from Thermo Fisher Scientific, Inc. Three different siRNAs were used to knockdown FoxO1 and si-FoxO1-1 was screened out as the most specific for subsequent experiments (Fig. S1). The cells were cultured with trypsin for $1 \mathrm{~h}$, and then the cells were transfected according to the instructions of the Lipofectamine ${ }^{\mathrm{TM}} 3000$ Transfection kit. Subsequently, the cells were cultured in an incubator with $5 \% \mathrm{CO}_{2}$ at $37^{\circ} \mathrm{C}$ for $48 \mathrm{~h}$. Meanwhile, the transfection efficiency of miR-124-5p-mimic/inhibitor was also detected by PCR (Fig. S2).

Enzyme-linked immunosorbent assay (ELISA). Human IL-6 ELISA kit (cat. no. ab178013; Abcam), Human TNF alpha ELISA kit (cat. no. ab181421; Abcam) and Human IL-8 ELISA kit (cat. no. ab214030; Abcam) were used to detect the levels of inflammatory cytokines including interleukin-6 (IL-6), interleukin-8 (IL-8) and tumor necrosis factor- $\alpha$ (TNF- $\alpha)$. According to the instructions of the kits, $50 \mu \mathrm{l}$ samples or standards were added to the appropriate wells of 96-well plates that were incubated with antibodies cocktail for $1 \mathrm{~h}$ at $37^{\circ} \mathrm{C}$. Then, each well was washed by $350 \mu \mathrm{l} 1 \mathrm{X}$ Wash Buffer PT for 3 times. Next, $100 \mu \mathrm{l}$ tetramethylbenzidine development solution were added into each well and incubated for $10 \mathrm{~min}$ in the dark. Finally, the results were detected by a Microplate Reader at $450 \mathrm{~nm}$ after the color reaction stopped. Each sample was assayed using three replicates.

Western blotting. The expression of nuclear factor $(\mathrm{NF})-\kappa \mathrm{B}$, intercellular adhesion molecule (ICAM)-1 and FoxO1 were detected by western blotting. The HUVECs were lysed by RIPA total protein lysate $(150 \mathrm{mM} \mathrm{NaCl}, 0.5 \%$ sodium deoxycholate, $50 \mathrm{mM}$ Tris- $\mathrm{HCl}, \mathrm{pH} 7.4,1 \% \mathrm{NP}-40)$ to obtain protein samples. The BCA protein quantitative detection kit was used to determine the protein concentration, according to the instructions. Firstly, the protein standard solution was prepared and added to the 96-well plates according to the gradient concentration, and the appropriate concentration of sample protein was added. Next, BCA solution $(A: B=50: 1)$ was added to the protein solution and incubated at $37^{\circ} \mathrm{C}$ for $30 \mathrm{~min}$. Finally, the absorbance was measured and the standard curve was drawn to determine the concentration of sample protein. Next, $40 \mu \mathrm{g}$ protein of each group was loaded into $10 \%$ SDS-PAGE gel, and GAPDH was used as the internal control to carry out concentrated gel electrophoresis and separation gel electrophoresis. Then, the transmembrane electrophoresis was implemented by using the polyvinylidene 

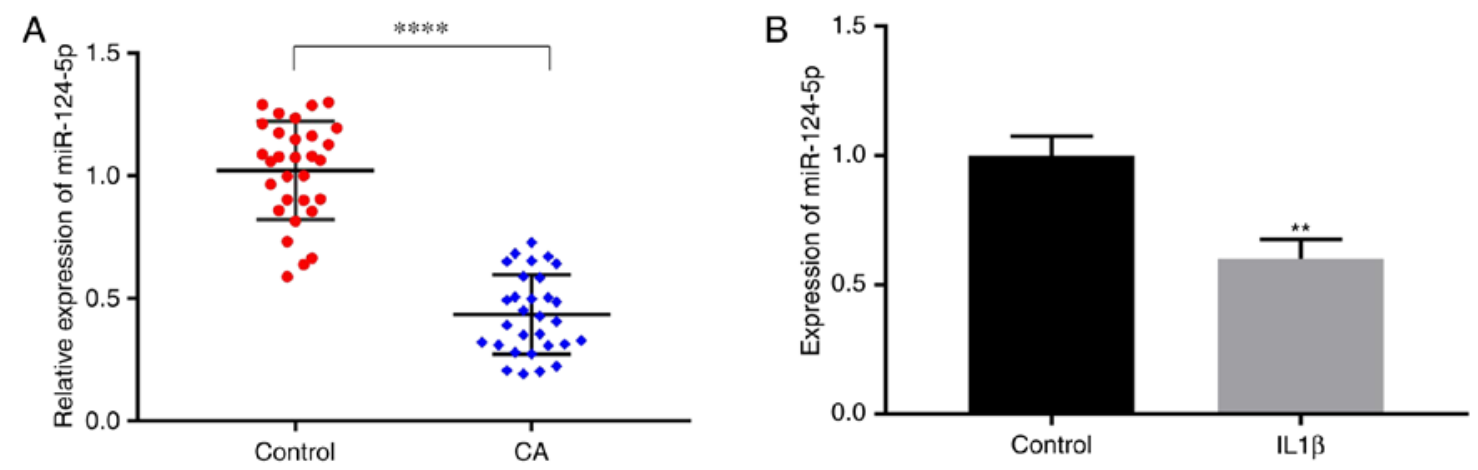

Figure 1. Downregulation of miR-124-5p in cerebral aneurysm in vivo and in vitro. (A) The expression of miR-124-5p in the peripheral blood of 30 patients with CA and 30 normal individuals was detected by RT-qPCR. (B) The expression of miR-124-5p in HUVECs stimulated by IL-1 $\beta$ and normal HUVECs was detected by RT-qPCR. ${ }^{* * *} \mathrm{P}<0.01 ;{ }^{* * * * *} \mathrm{P}<0.0001$ vs. control. miR, microRNA; RT-qPCR, reverse transcription-quantitative PCR; IL-1 $\beta$, interleukin-1 $\beta$.

fluoride (PVDF) membrane. The PVDF membrane was blocked with $5 \%$ bovine serum albumin (BSA) for $1 \mathrm{~h}$ at $37^{\circ} \mathrm{C}$. The membrane was then incubated with primary antibodies purchased from Abcam, including anti-NF-NF- $\mathrm{BB}$ (cat. no. ab16502; $0.5 \mu \mathrm{g} / \mathrm{ml}$ ), anti-ICAM-1 (cat. no. ab109361; 1:10,000), anti-FoxO1 (cat. no. ab70382; 1:10,000) and anti-GADPH (cat. no. ab9485; 1/12,500) antibodies, for $14 \mathrm{~h}$ at $4^{\circ} \mathrm{C}$. Subsequently, the secondary antibody Goat Anti-Rabbit IgG H\&L (HRP) (cat. no. ab205718; Abcam; 1:50,000) was added to the PVDF membranes and incubated for $90 \mathrm{~min}$ at $37^{\circ} \mathrm{C}$. Eventually, the PVDF membrane was put into the gel imager with ECL luminescent reagent for exposure for $30 \mathrm{sec}$. The proteins were quantified using ImageJ software (v1.8.0; National Institutes of Health).

Dual luciferase reporter assay. The binding site of FoxO1 in miR-124-5p was predicted using the TargetScan website (http://www.targetscan.org/mmu_72/), and the double luciferase reporter gene analysis was applied to identify the targeting association between miR-124-5p and FoxO1. The FoxO1 3'-UTR containing the miR-124-5p binding sites was synthesized and inserted into the pGL3 control vector between the BamH1 site and Xhal site to construct the FoxO1 wild-type (FoxO1-WT) reporter vector. The FoxO1-WT reporter vector $(100 \mathrm{ng})$ and miR-124-5p mimics $(50 \mathrm{nM})$ or mimic-NC $(100 \mathrm{nM})$ were co-transfected into the cells at $37^{\circ} \mathrm{C}$ for $8 \mathrm{~h}$ using Lipofectamine ${ }^{\mathrm{TM}} 3000$ Transfection kit. The aforementioned process was also applied to the corresponding FoxO1 mutant (FoxO1 MUT) reporter vector. The activity of luciferase was determined by the Dual-Luciferase Reporter Assay System kit (cat. no. E1910; Shanghai Haoran Biotechnology Co., Ltd.) after transfection for $48 \mathrm{~h}$.

Transwell migration/invasion assay. The migration and invasion abilities of the HUVECs were detected by using Transwell chambers. After digestion and washing, the cells at the logarithmic growth stage were resuspended in serum-free medium at a cell concentration of $2 \times 10^{5}$ cells $/ \mathrm{ml}$. Next, $650 \mu \mathrm{l}$ medium containing $10 \%$ FBS was added to the lower chamber, $150 \mu \mathrm{l}$ cell suspension was added to the upper chamber, and the chamber was incubated for $24 \mathrm{~h}$ at $37^{\circ} \mathrm{C}$. Then, the cells on the upper surface of the membrane were removed slowly using a cotton swab. Subsequently, the cells on the lower surface of the membrane were fixed with $4 \%$ methanol for $30 \mathrm{~min}$ at room temperature after the membrane was dried, and the cells were stained by Giemsa for $25 \mathrm{~min}$ at room temperature. Ultimately images were captured with a Leica DC $300 \mathrm{~F}$ light microscope (magnification, $\mathrm{x} 400$ ) and the cells were counted using the ImageJ software (v1.8.0; National Institutes of Health) to assess the ability of cell migration. The invasion assay was performed as aforementioned for the migration assay, except that Matrigel gel diluted by serum-free DMEM medium was added to the upper chamber.

Statistical analysis. All experiments were repeated three times, and all data are presented as means \pm SD. The data were evaluated using statistical approach, including one-way ANOVA followed by Sidak's multiple comparisons test or Student's t-test, using the GraphPad Prism 7.0 software (GraphPad Software, Inc.). P $<0.05$ was considered to indicate a statistically significant difference.

\section{Results}

Downregulation of miR-124-5p in CA in vivo and in vitro. The difference in the expression of miR-124-5p in the peripheral blood of 30 patients with CA and 30 healthy individuals was analyzed by using RT-qPCR. The results showed that the expression of miR-124-5p was lower in patients with CA compared with that of healthy individuals (Fig. 1A). In addition, according to previous studies, It is known that IL-1 $\beta$ can activate vascular endothelial cells and induce their secretion of adhesion factors such as ICAM-1, in order to recruit inflammatory cells and trigger the inflammatory reaction of arterial walls, thus forming aneurysm (32). In the present study, HUVECs were used as an in vitro model system, and the IL-1 $\beta$ were used to stimulate HUVECs to simulate the inflammatory state of CA. Then, the expression of miR-124-5p was detected in HUVECs that were simulated by IL-1 $\beta$ and the non-stimulated HUVECs using RT-qPCR. The results showed that the expression of miR-124-5p in the IL-1 $\beta$ group was significantly lower compared with that of the control group (Fig. 1B).

Inhibition of miR-124-5p can enhance the cell function of HUVECs and promote the release of inflammatory 
A

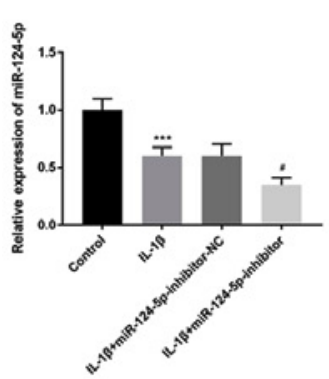

B

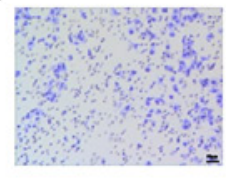

Control

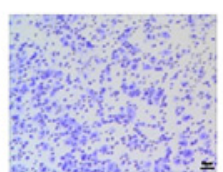

IL-1 $\beta$

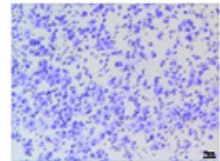

IL-1 $\beta+$ miR-124 -5p-inhibitor-NC

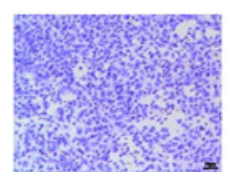

IL-1 $\beta+$ miR-124

$-5 p$-inhibitor
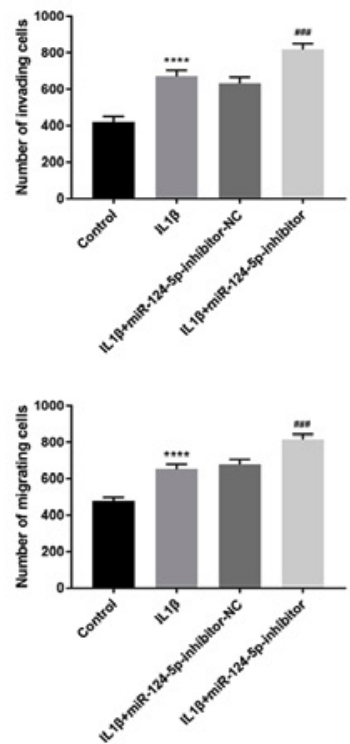

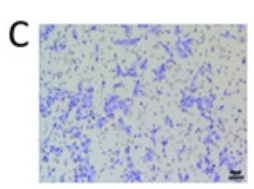

Control

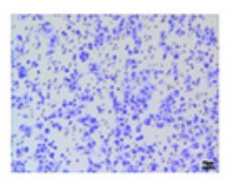

IL-1 $\beta$

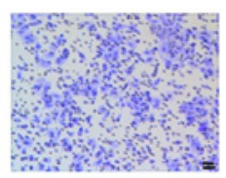

IL-1 $\beta+$ miR-124

-5p-inhibitor-NC

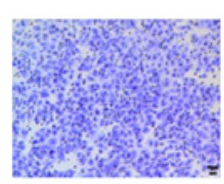

IL- $1 \beta+$ miR-124

$-5 p$-inhibitor

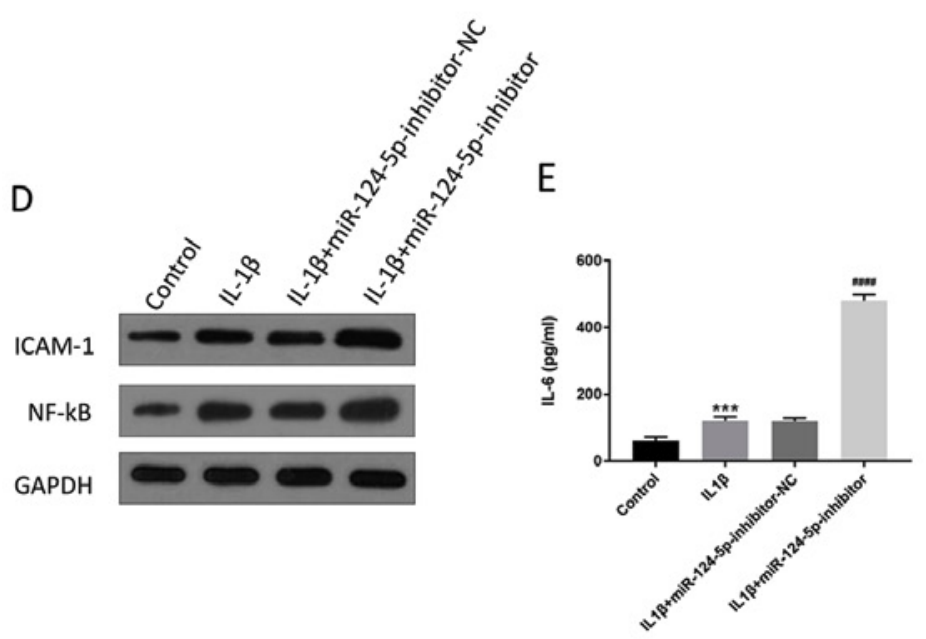

$\mathrm{F}$

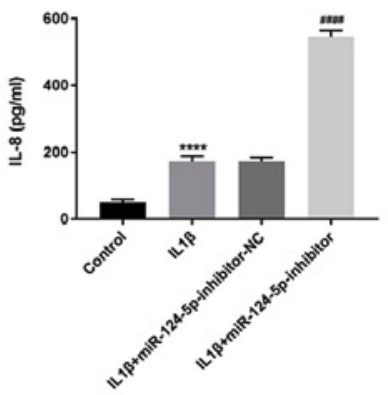

G

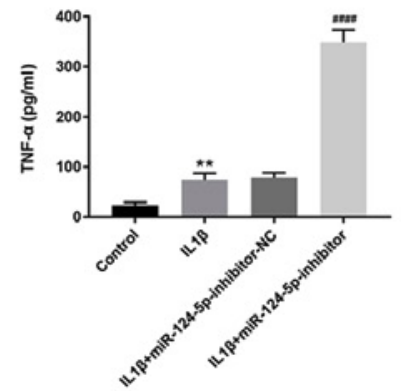

Figure 2. Inhibition of miR-124-5p can enhance the cell function of HUVECs and promote the release of inflammatory factors. In this group of experiment, HUVECs were treated differently and divided into four groups: Control group; IL-1 $\beta$ group; IL-1 $\beta+$ miR-124-5p-inhibitor-NC; IL-1 $\beta+$ miR-124-5p-inhibitor. The transfection of miR-124-5p-inhibitor decreased the expression of miR-124-5p, and miR-124-5p-inhibitor-NC was the negative control. (A) The changes in miR-124-5p expression in HUVECs were detected by reverse transcription-quantitative PCR. The (B) invasion and (C) migration abilities of HUVECs were detected by Transwell assay. Scale bar, $50 \mu \mathrm{m}$. (D) The changes in the expression of ICAM-1 and NF- $\mathrm{BB}$ in HUVECs were detected by western blotting. GAPDH was used as the internal reference. The expression of (E) IL-6, (F) IL-8 and (G) TNF- $\alpha$ in HUVECs was detected by enzyme-linked immu-

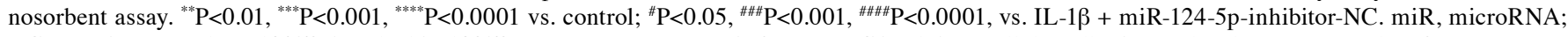
$\mathrm{NC}$, negative control; IL-1 $\beta / 6 / 8$, interleukin- $1 \beta / 6 / 8$; TNF- $\alpha$, tumor necrosis factor- $\alpha$; ICAM1, intercellular adhesion molecule; NF- $\kappa \mathrm{B}$, nuclear factor- $\kappa \mathrm{B}$.

factors. The changes in the cell function of HUVECs and the associated inflammatory factors were detected following the downregulation of miR-124-5p by the miR-124-5p-inhibitor. The results of RT-qPCR showed that IL-1 $\beta$ could inhibit the expression of miR-124-5p, and there was no significant difference between the IL-1 $\beta$ group and IL-1 $\beta+$ miR-124-5p-inhibitor-negative control (NC) group (Fig.2A).Furthermore, the application of miR-124-5p-inhibitor could enhance the inhibitory effect of miR-124-5p compared with the IL-1 $\beta+$ miR-124-5p-inhibitor-NC group (Fig. 2A). The results of the cell invasion assay indicated that the IL-1 $\beta$ group had higher invasion ability compared with the control group, and the invasion ability was further enhanced by miR-124-5p-inhibitor compared with the miR-124-5p-inhibitor-NC (Fig. 2B). Compared with the control group, the migration ability of HUVECs was strengthened by IL-1 $\beta$, and miR-124-5p-inhibitor further promoted the cell migration compared with the IL-1 $\beta+$ miR-124-5p-inhibitor-NC group (Fig. 2C). Meanwhile, western blotting and ELISA were used to detect the expression of inflammatory factors. Western blotting showed that the expression of $\mathrm{NF}-\kappa \mathrm{B}$ and ICAM-1 was increased in the IL-1 $\beta$ group compared with the control group, and the transfection of miR-124-5p-inhibitor enhanced the expression of inflammatory factors compared with the IL-1 $\beta+$ miR-124-5p-inhibitor-NC group (Fig. 2D). The results of ELISA indicated that IL-1 $\beta$ upregulated the expression levels of IL-6, IL- 8 and TNF- $\alpha$ compared with the control group, and the miR-124-5p-inhibitor further promoted the increase in the expression of inflammatory factors (Fig. 2E-G). The aforementioned experimental results demonstrate that the inhibition of miR-124-5p could enhance the cellular function of HUVECs and promote the release of inflammatory factors. 


\section{A FOXO1 3'UTR 5'-CUAAAUGUUUAACAAGAACACAA-3' FOXO1 3UTR 5'-CUAAAUGUUUAACAAGAACACAA- hsa-miR-124-5p 3'-UAGUUCCAGGCGACACUUGUGC-5'}
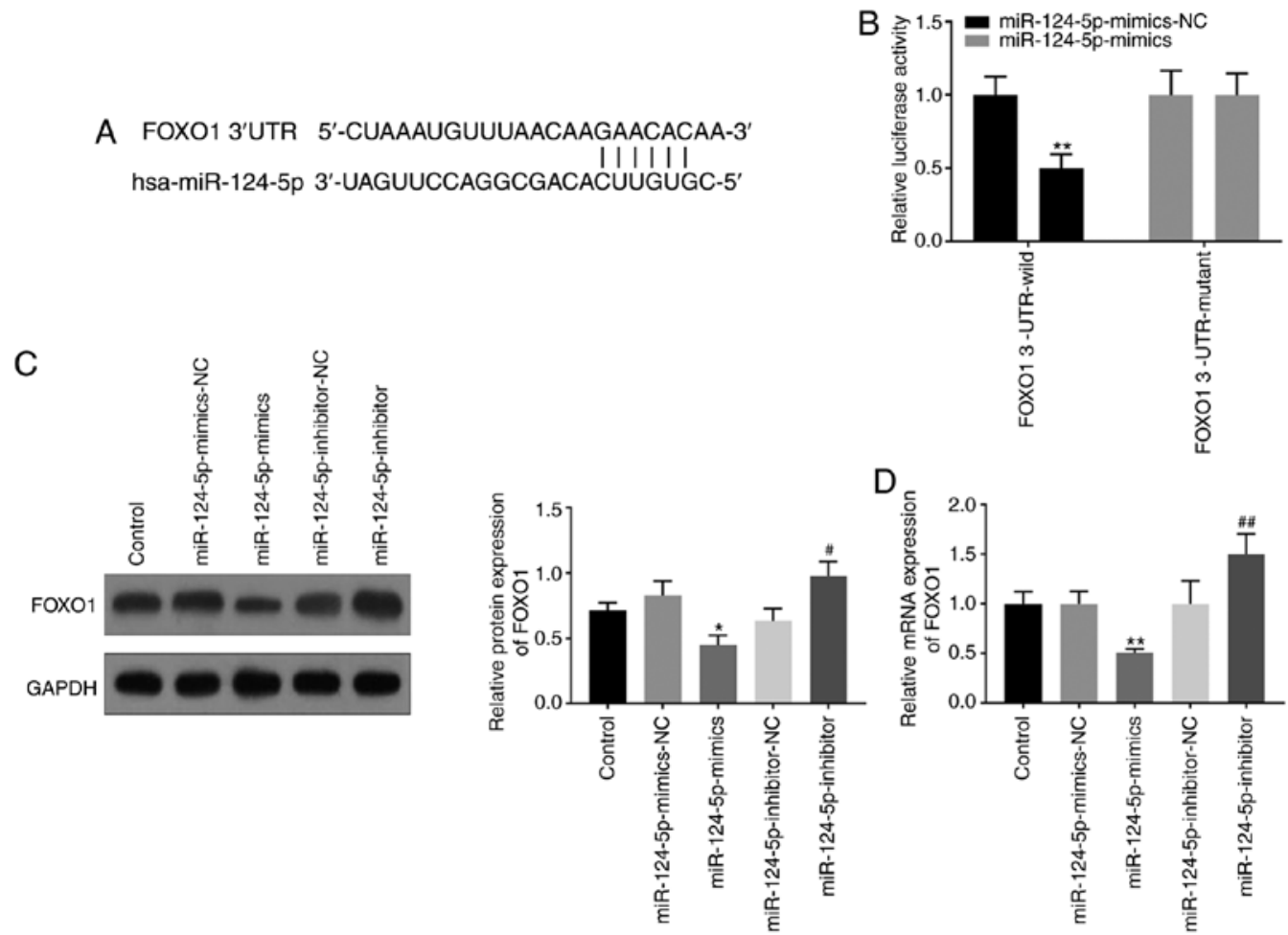

Figure 3. FoxO1 is the target gene of miR-124-5p. (A) The binding sites of miR-124-5p and FoxO1 obtained from the TargetScan website. (B) Dual luciferase reporter assay was used to detect whether wild-type-FoxO1 is the target gene of miR-124-5p, and the mutant-FoxO1 was the control. miR-124-5p-mimics were used to simulate the high-level expression of endogenous mature miR-124-5p in cells, and the miR-124-5p-mimics-NC was the negative control. (C) Western blotting was applied to detect whether the expression of FoxO1 in HUVECs changed with the expression of miR-124-5p, and the quantitative analysis of protein bands was also implemented by using Image J. GAPDH was used as the internal reference. (D) The changes in relative mRNA level of FoxO1 was detected by reverse transcription-quantitative PCR. ${ }^{*} \mathrm{P}<0.05,{ }^{* *} \mathrm{P}<0.01$ vs. miR-124-5p-mimics-NC; ${ }^{\#} \mathrm{P}<0.05,{ }^{\# \#} \mathrm{P}<0.01$ vs. miR-124-5p-inhibitor-NC. miR, microRNA; $\mathrm{NC}$, negative control; UTR, untranslated region.

FoxOl is the target gene of miR-124-5p. It was predicted that FoxO1 is the target gene of miR-124-5p by using the TargetScan website (Fig. 3A); this was verified using dual luciferase reporter and western blotting assays. The results of the dual luciferase assay showed decreased luciferase activity in the FoxO1 3'-UTR wild-type group, following transfection with miR-124-5p-mimics, compared with the miR-124-5p-mimics-NC group, but there was no significant change in the FoxO1 3'-UTR mutant group (Fig. 3B). Results of western blotting displayed that the miR-124-5p-inhibitor increased the expression of FoxO1, while miR-124-5p-mimics decreased the expression of FoxO1 (Fig. 3C). The results of RT-qPCR also showed that the miR-124-5p-mimics group had lower expression of FoxO1 compared with the control group, and the miR-124-5p-inhibitor group had the opposite results (Fig. 3D). All the experimental results indicated that FoxO1 was the target gene of miR-124-5p, and miR-124-5p could negatively regulate FoxO1.

miR-124-5p regulates the cell migration and invasion, as well as the release of inflammatory factors, through mediating FoxOl expression. A series of experiments were conducted to verify the interaction of FoxO1 with miR-124-5p in HUVECs. The results of western blotting showed that the upregulated expression of FoxO1 induced by miR-124-5p-inhibitor was weakened by the si-FoxO1 (Fig. 4A). The results of cell invasion and migration experiments indicated that inhibition of miR-124-5p promoted the invasion and migration of HUVECs, which was weakened after the cells were co-transfected with miR-124-5p-inhibitor and si-FoxO1 (Fig. 4B and C). At the same time, the increase in NF- $\mathrm{B}$, ICAM-1, IL-6, IL-8 and TNF- $\alpha$ that was induced by miR-124-5p-inhibitor were downregulated by FoxO1 knockdown (Fig. 4A, D-F). All the experimental results showed that inhibition of miR-124-5p promoted the cell function and the release of inflammatory factors of HUVECs, which could be reversed by FoxO1 knockdown. Hence, miR-124-5p regulated the cell function and the release of inflammatory factors of HUVECs through mediating FoxO1 expression.

\section{Discussion}

The present study demonstrated that the expression of miR-124-5p in patients with CA and in HUVECs that were stimulated by IL-1 $\beta$ was lower compared with that in healthy individuals or cells. The lower expression of miR-124-5p could promote the ability of cell invasion, migration and the inflammatory response of HUVECs. Then, FoxO1 was predicted as a target gene of miR-124-5p. The inhibition of miR-124-5p promoted the cell migration, cell invasion and the release of inflammatory factors, which could be reversed by FoxO1 knockdown. Hence, it was speculated that miR-124-5p could delay the progression of CA by inhibiting the expression of FoxO1.

The environmental and genetic factors of the formation of $\mathrm{CA}$ have been reported, but the pathological factors 

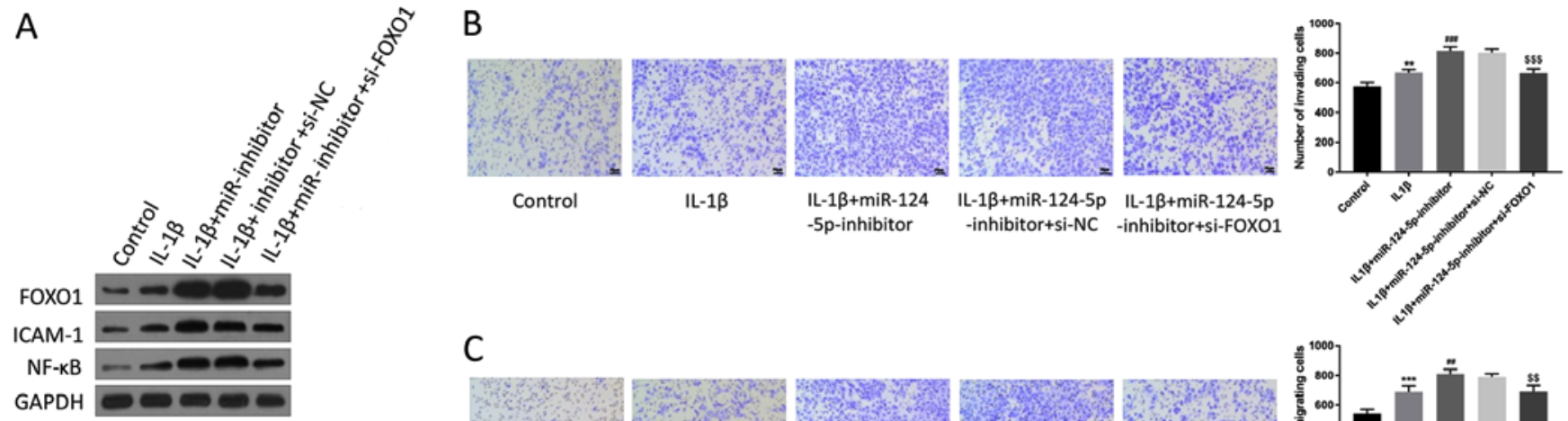

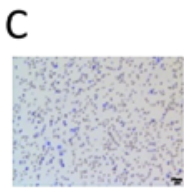

Control

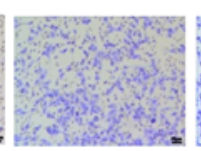

IL-1 $\beta$

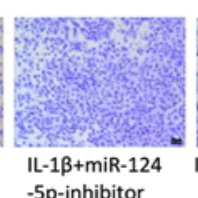

-5p-inhibitor
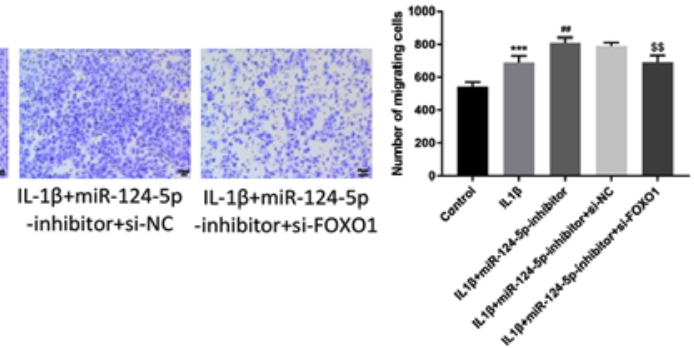

D

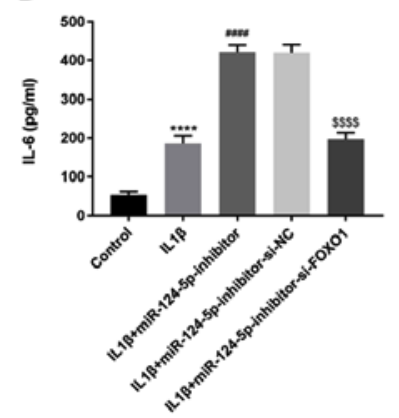

$E$

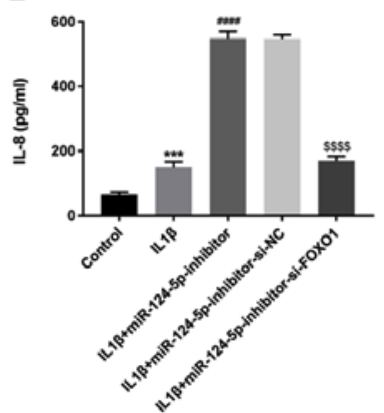

$\mathrm{F}$

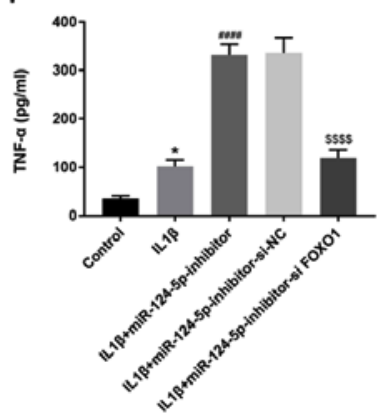

Figure 4. miR-124-5p regulates cell migration and invasion, as well as the release of inflammatory factors, through mediating FoxO1 expression. (A) The changes in the expression levels of FoxO1, ICAM-1 and NF- $\mathrm{B}$ in HUVECs after transfecting with siRNA-FoxO1 was detected by western blotting. GAPDH was used as the internal reference. The changes in (B) invasion and (C) migration abilities of HUVECs were detected by using Transwell assays after FoxO1 knockdown. Scale bar, $50 \mu \mathrm{m}$. The changes in (D) IL-6, (E) IL-8 and (F) TNF- $\alpha$ of HUVECs were detected by enzyme-linked immunosorbent assay after FoxO1 knockdown. ${ }^{*} \mathrm{P}<0.05,{ }^{* *} \mathrm{P}<0.01,{ }^{* * *} \mathrm{P}<0.001,{ }^{* * * * *} \mathrm{P}<0.0001$ vs. control; ${ }^{\# \#} \mathrm{P}<0.01,{ }^{\# \#} \mathrm{P}<0.001,{ }^{\# \# \# \#} \mathrm{P}<0.0001$ vs. IL- $\beta ;{ }^{\$ \$} \mathrm{P}<0.01,{ }^{\$ \$ \$} \mathrm{P}<0.001,{ }^{\$ \$ \$ \$} \mathrm{P}<0.0001$ vs. IL-1 $\beta+$ miR-124-5p-Inhibitor-si-NC. miR, microRNA; NC, negative control; TNF- $\alpha$, tumor necrosis factor- $\alpha$; ICAM1, intercellular adhesion molecule; $\mathrm{NF}-\kappa \mathrm{B}$, nuclear factor- $\kappa \mathrm{B}$; siRNA/si-, small interfering RNA; IL-6/8, interleukin-6/8.

of its occurrence and development have not been fully acquainted (33). Furthermore, the most recognized factors that promote the development of $\mathrm{CA}$ are inflammation and hemodynamics (34). NF- $\mathrm{NB}$ is one of the key components to regulate the expression of proinflammatory genes and it induces the expression of proinflammatory cytokines, chemokines and adhesion molecules (35). It has been reported that the expression of $\mathrm{NF}-\kappa \mathrm{B}$ in $\mathrm{CA}$ is significantly higher compare with normal tissues and the activation of $\mathrm{NF}-\kappa \mathrm{B}$ is a risk factor in the occurrence and development of CA (36-38). In inflammatory reaction, endotoxin and some chemokines can promote the expression of ICAM-1 in endothelial cells and leukocytes in the wall of the porch (39). Furthermore, the activated leukocytes adhere to the vascular wall by ICAM-1, and then pass through the vessel wall and enter the focus of infection (39). TNF- $\alpha$ plays a role in vascular inflammation when the vascular inflammation is closely associate with the progression of aneurysm (40-42). Some studies have confirmed that hemodynamic stress increases the expression of TNF- $\alpha$, and TNF- $\alpha$ inhibitors can inhibit the formation of aneurysm and delay the disease process of aneurysm $(42,43)$. Studies have shown that cytokines such as IL-6 and IL-8 can downregulate procollagen biosynthesis at the transcription level, while the decrease in arterial wall collagen is a significant pathological feature of CA (44). Since the aforementioned inflammatory factors are closely associated with the occurrence and process of $\mathrm{CA}$, the present study examined the changes in these inflammatory factors under various experimental treatments.

Previous studies have shown that miRNAs can affect multiple signaling pathways to regulate the cell function, such as the ability of migration and invasion of cancer cells (45-48). However, according to the research of miR-124-5p in diseases, the effect of miR-124-5p on the ability of migration and invasion for glioblastoma multiforme cells is not obvious (27). Because the influence of miR-124-5p on the cell function of CA cells has not been studied, the present study attempted to study about CA by using HUVECs. The results indicated that the expression level of miR-124-5p in patients with CA or in cells was lower compared with that in normal individuals or cells. It was also found that the miR-124-5p inhibitor increased the ability of cell migration and invasion of HUVECs stimulated by IL-1 $\beta$. It has been reported that miRNAs can regulate the expression of a variety of inflammatory factors; for example, Wu et al (49) found that miR-124-5p can relieve the cerebral injury that is caused by inflammation by downregulating the 
expression of IL-6 and TNF- $\alpha$. The present study further indicated that the decrease in miR-124-5p could increase the release of inflammatory factors such as IL-6, IL-8, TNF- $\alpha$, ICAM-1 and NF- $\kappa \mathrm{B}$.

In order to further explore the molecular mechanism of miR-124-5p in CA, target gene FoxO1 of miR-124-5p was identified by using the TargetScan website. Previous studies have shown that FoxO1 knockdown can inhibit inflammatory response; for example, Malik et al (50) found that FoxO1 knockdown can decrease the expression of IL-1 $\beta$, thus, relieving the symptoms of diabetic retinopathy, and Wu et al (51) found that the allergic inflammation in asthmatic patients can be lowered by the decrease in IL-9 that is regulated by knocking down FoxO1. The present results indicated that inhibition of $\mathrm{miR}-124-5 \mathrm{p}$ enhanced the release of inflammatory factors (IL-6, IL-8, TNF- $\alpha, N F-\kappa B$ and ICAM-1), which was reversed by FoxO1 knockdown. Thus, the present study showed the inhibition on the inflammation caused by FoxO1 knockdown is in agreement with the previous reports. Furthermore, the promotion of migration and invasion of HUVECs induced by miR-124-5p inhibitors was weakened by the introduction of FoxO1 siRNA. Therefore, miR-124-5p regulated the migration and invasion abilities and the release of inflammatory factors of HUVECs through mediating FoxO1 expression.

The present study focused on the regulation of miR-124-5p and FoxO1 on the cellular function and the release of inflammatory factors by HUVECs in vitro, however, whether miR-124-5p/FoxO1 pathway is also associated with inflammatory process or cellular function in CA animal models is the focus of the follow-up study. In addition, whether factors (such as matrix metalloproteinases) associated with CA in vivo or in vitro are regulated by miR-124-5p/FoxO1 pathway will also be studied in the future.

In conclusion, miR-124-5p was able to inhibit the cellular function and the release of inflammatory factors of HUVECs by downregulating the expression of FoxO1, which might prevent the occurrence of CA or delay the development of CA. Therefore, the present results provide the potential biomarkers and signaling pathway for the treatment of CA, and supply another way to explore the molecular mechanism of the occurrence and development of CA.

\section{Acknowledgements}

Not applicable.

\section{Funding}

No funding was received.

\section{Availability of data and materials}

All data generated or analyzed during this study are included in this published article.

\section{Authors' contributions}

RKW and GYY designed experiments; YYS, GYL and HTY collected data; XJL, KFL and XZ analyzed experimental results. RKW wrote the manuscript; GYY approved the manuscript. All authors read and approved the final manuscript.

\section{Ethics approval and consent to participate}

The institutional ethics committee of Handan Central Hospital has approved this study. Written informed consent was obtained from all participants and their families.

\section{Patient consent for publication}

Not applicable.

\section{Competing interests}

The authors declare that they have no competing interests.

\section{References}

1. Cebral JR and Raschi M: Suggested connections between risk factors of intracranial aneurysms: A review. Ann Biomed Eng 41: 1366-1383, 2013

2. Sadasivan C, Fiorella DJ, Woo HH and Lieber BB: Physical factors effecting cerebral aneurysm pathophysiology. Ann Biomed Eng 41: 1347-1365, 2013.

3. Kataoka H: Molecular mechanisms of the formation and progression of intracranial aneurysms. Neurol Med Chir (Tokyo) 55: 214-229, 2015.

4. Alg VS, Sofat R, Houlden H and Werring DJ: Genetic risk factors for intracranial aneurysms: A meta-analysis in more than 116,000 individuals. Neurology 80: 2154-2165, 2013.

5. Juvela S: Prevalence of and risk factors for intracranial aneurysms. Lancet Neurol 10: 595-597, 2011.

6. Chalouhi N, Hoh BL and Hasan D: Review of cerebral aneurysm formation, growth, and rupture. Stroke 44: 3613-3622, 2013.

7. Wei L, Wang Q, Zhang Y, Yang C, Guan H, Chen Y and Sun Z: Identification of key genes, transcription factors and microRNAs involved in intracranial aneurysm. Mol Med Rep 17: 891-897, 2018.

8. Wang WH, Wang YH, Zheng LL, Li XW, Hao F and Guo D: MicroRNA-29a: A potential biomarker in the development of intracranial aneurysm. J Neurol Sci 364: 84-89, 2016.

9. Hosaka K and Hoh BL: Inflammation and cerebral aneurysms. Transl Stroke Res 5: 190-198, 2014.

10. Kanematsu Y, Kanematsu M, Kurihara C, Tada Y, Tsou TL, van Rooijen N, Lawton MT, Young WL, Liang EI, Nuki Y and Hashimoto T: Critical roles of macrophages in the formation of intracranial aneurysm. Stroke 42: 173-178, 2011.

11. Aoki T, Kataoka H, Ishibashi R, Nozaki K, Egashira K and Hashimoto N: Impact of monocyte chemoattractant protein-1 deficiency on cerebral aneurysm formation. Stroke 40: 942-951, 2009.

12. Aoki T, Kataoka H, Nishimura M, Ishibashi R, Morishita R and Miyamoto S: Ets-1 promotes the progression of cerebral aneurysm by inducing the expression of MCP-1 in vascular smooth muscle cells. Gene Ther 17: 1117-1123, 2010.

13. Aoki T, Yamamoto K, Fukuda M, Shimogonya Y, Fukuda S and Narumiya S: Sustained expression of MCP-1 by low wall shear stress loading concomitant with turbulent flow on endothelial cells of intracranial aneurysm. Acta Neuropathol Commun 4: 48, 2016.

14. Bartel DP: MicroRNAs: Target recognition and regulatory functions. Cell 136: 215-233, 2009.

15. Chen X, Zhao Y, Wang F, Bei Y, Xiao J and Yang C: MicroRNAs in liver regeneration. Cell Physiol Biochem 37: 615-628, 2015.

16. Ameres SL and Zamore PD: Diversifying microRNA sequence and function. Nat Rev Mol Cell Biol 14: 475-488, 2013.

17. Pal AS and Kasinski AL: Animal models to study microRNA function. Adv Cancer Res 135: 53-118, 2017.

18. Henninger $\mathrm{N}$ and Mayasi Y: Nucleic acid therapies for ischemic stroke. Neurotherapeutics 16: 299-313, 2019.

19. Martinez B and Peplow PV: Blood microRNAs as potential diagnostic and prognostic markers in cerebral ischemic injury. Neural Regen Res 11: 1375-1378, 2016. 
20. Chu-Tan JA, Rutar M, Saxena K, Aggio-Bruce R, Essex RW, Valter K, Jiao H, Fernando N, Wooff Y, Madigan MC, et al: MicroRNA-124 dysregulation is associated with retinal inflammation and photoreceptor death in the degenerating retina. Invest Ophthalmol Vis Sci 59: 4094-4105, 2018.

21. Kawano $S$ and Nakamachi Y: miR-124a as a key regulator of proliferation and MCP-1 secretion in synoviocytes from patients with rheumatoid arthritis. Ann Rheum Dis 70 (Suppl 1): i88-i91, 2011.

22. Sun Y, Luo ZM, Guo XM, Su DF and Liu X: An updated role of microRNA-124 in central nervous system disorders: A review. Front Cell Neurosci 9: 193, 2015.

23. Dong N, Xu B, Shi $\mathrm{H}$ and Lu Y: miR-124 regulates amadori-glycated albumin-induced retinal microglial activation and inflammation by targeting Rac1. Invest Ophthalmol Vis Sci 57: 2522-2532, 2016.

24. Sun L, Zhao M, Zhang J, Lv M, Li Y, Yang X, Liu A and Wu Z: miR-29b downregulation induces phenotypic modulation of vascular smooth muscle cells: Implication for intracranial aneurysm formation and progression to rupture. Cell Physiol Biochem 41: 510-518, 2017.

25. Signorelli F, Sela S, Gesualdo L, Chevrel S, Tollet F, Pailler-Mattei C, Tacconi L, Turjman F, Vacca A and Schul DB: Hemodynamic stress, inflammation, and intracranial aneurysm development and rupture: A systematic review. World Neurosurg 115: 234-244, 2018

26. Wang Y, Huang C, Chintagari NR, Xi D, Weng T and Liu L: miR-124 regulates fetal pulmonary epithelial cell maturation. Am J Physiol Lung Cell Mol Physiol 309: L400-L413, 2015.

27. Chen Q, Lu G, Cai Y, Li Y, Xu R, Ke Y and Zhang S: miR-124-5p inhibits the growth of high-grade gliomas through posttranscriptional regulation of LAMB1. Neuro Oncol 16: 637-651, 2014.

28. Liu P, Shi Y, Fan Z, Zhou Y, Song Y, Liu Y, Yu G, An Q and Zhu W: Inflammatory smooth muscle cells induce endothelial cell alterations to influence cerebral aneurysm progression via regulation of integrin and VEGF expression. Cell Transplant 28: 713-722, 2019.

29. Livak KJ and Schmittgen TD: Analysis of relative gene expression data using real-time quantitative PCR and the 2(-Delta Delta C(T)) method. Methods 25: 402-408, 2001

30. Liang Y, Zhu D, Zhu L, Hou Y, Hou L, Huang X, Li L, Wang Y, Li L, Zou H, et al: Dichloroacetate overcomes oxaliplatin chemoresistance in colorectal cancer through the miR-543/PTEN/Akt/mTOR pathway. J Cancer 10: 6037-6047, 2019.

31. Tang Z, Gong Z and Sun X: LncRNA DANCR involved osteolysis after total hip arthroplasty by regulating FOXO1 expression to inhibit osteoblast differentiation. J Biomed Sci 25: 4, 2018.

32. Skaria T, Bachli E and Schoedon G: RSPO3 impairs barrier function of human vascular endothelial monolayers and synergizes with pro-inflammatory IL-1. Mol Med 24: 45, 2018.

33. Chalouhi N, Ali MS, Jabbour PM, Tjoumakaris SI, Gonzalez LF, Rosenwasser RH, Koch WJ and Dumont AS: Biology of intracranial aneurysms: Role of inflammation. J Cereb Blood Flow Metab 32: 1659-1676, 2012.

34. Tulamo R, Frösen J, Hernesniemi J and Niemelä M: Inflammatory changes in the aneurysm wall: A review. J Neurointerv Surg 2: 120-130, 2010.

35. Karin $M$ and Ben-Neriah Y: Phosphorylation meets ubiquitination: The control of NF-[kappa]B activity. Annu Rev Immunol 18: 621-663, 2000.

36. Catrysse L and van Loo G: Inflammation and the metabolic syndrome: The tissue-specific functions of NF- $\kappa \mathrm{B}$. Trends Cell Biol 27: 417-429, 2017.
37. Cheng WT and Wang N: Correlation between MMP-2 and NF- $\kappa$ B expression of intracranial aneurysm. Asian Pac J Trop Med 6: 570-573, 2013.

38. Aoki T, Kataoka H, Nishimura M, Ishibashi R, Morishita R and Miyamoto $\mathrm{S}$ : Regression of intracranial aneurysms by simultaneous inhibition of nuclear factor- $\kappa \mathrm{B}$ and Ets with chimeric decoy oligodeoxynucleotide treatment. Neurosurgery 70: 1534-1543, 2012

39. Sun W, Watanabe $Y$ and Wang ZQ: Expression and significance of ICAM-1 and its counter receptors LFA-1 and Mac-1 in experimental acute pancreatitis of rats. World J Gastroenterol 12: 5005-5009, 2006.

40. Yagi K, Tada Y, Kitazato KT, Tamura T, Satomi J and Nagahiro S: Ibudilast inhibits cerebral aneurysms by down-regulating inflammation-related molecules in the vascular wall of rats. Neurosurgery 66: 551-559, 2010.

41. Ali MS, Starke RM, Jabbour PM, Tjoumakaris SI, Gonzalez LF, Rosenwasser RH, Owens GK, Koch WJ, Greig NH and Dumont AS: TNF- $\alpha$ induces phenotypic modulation in cerebral vascular smooth muscle cells: Implications for cerebral aneurysm pathology. J Cereb Blood Flow Metab 33: 1564-1573, 2013.

42. Starke RM, Chalouhi N, Jabbour PM, Tjoumakaris SI, Gonzalez LF, Rosenwasser RH, Wada K, Shimada K, Hasan DM, Greig NH, et al: Critical role of TNF- $\alpha$ in cerebral aneurysm formation and progression to rupture. J Neuroinflammation 11: 77, 2014.

43. Yokoi T, Isono T, Saitoh M, Yoshimura Y and Nozaki K: Suppression of cerebral aneurysm formation in rats by a tumor necrosis factor- $\alpha$ inhibitor. J Neurosurg 120: 1193-1200, 2014.

44. Aoki T, Kataoka H, Ishibashi R, Nozaki K, Morishita R and Hashimoto N: Reduced collagen biosynthesis is the hallmark of cerebral aneurysm: Contribution of interleukin-1beta and nuclear factor-kappaB. Arterioscler Thromb Vasc Biol 29: 1080-1086, 2009.

45. Garofalo M and Croce CM: MicroRNAs: Master regulators as potential therapeutics in cancer. Annu Rev Pharmacol Toxicol 51: 25-43, 2011.

46. Guo X, Guo L, Ji J, Zhang J, Zhang J, Chen X, Cai Q, Li J, Gu Q, Liu B, et al: miRNA-331-3p directly targets E2F1 and induces growth arrest in human gastric cancer. Biochem Biophys Res Commun 398: 1-6, 2010.

47. Martello G, Rosato A, Ferrari F, Manfrin A, Cordenonsi M, Dupont S, Enzo E, Guzzardo V, Rondina M, Spruce T, et al: A microRNA targeting dicer for metastasis control. Cell 141: 1195-1207, 2010.

48. Guo X, Jing C, Li L, Zhang L, Shi Y, Wang J, Liu J and Li C: Down-regulation of VEZT gene expression in human gastric cancer involves promoter methylation and miR-43c. Biochem Biophys Res Commun 404: 622-627, 2011.

49. Wu Y, Yao J and Feng K: miR-124-5p/NOX2 axis modulates the ROS production and the inflammatory microenvironment to protect against the cerebral I/R injury. Neurochem Res 45: 404-417, 2020

50. Malik S, Sadhu S, Elesela S, Pandey RP, Chawla AS, Sharma D, Panda L, Rathore D, Ghosh B, Ahuja V and Awasthi A: Transcription factor Foxol is essential for IL-9 induction in T helper cells. Nat Commun 8: 815, 2017.

51. Wu L, Guo F, Wu Y, Wang Q, Ma X, Zhao Y and Qin G: The role of FoxO1 in interleukin-1 $\beta$-induced autostimulation in retina endothelial cells and retinas of diabetic rats. Microvasc Res 112: 93-100, 2017.

This work is licensed under a Creative Commons Attribution-NonCommercial-NoDerivatives 4.0 International (CC BY-NC-ND 4.0) License. 